\title{
Cryogenic instrumentation at ProtoDUNE
}

\author{
M. Á. García-Peris ${ }^{a, *}$ for the DUNE Collaboration \\ ${ }^{a}$ Instituto de Física Corpuscular (Universitat de Valencia-CSIC), \\ Catedratico Jose Beltran, 2 E-46980 Paterna (Valencia), Spain \\ E-mail: migarpez@ific.uv.es
}

The cryogenic instrumentation of ProtoDUNE successfully operated during the first run of the experiment, providing a comprehensive monitoring of the cryostats, the cryogenics system and the detectors. It includes devices to measure the liquid argon level, the electron lifetime, the temperature of the liquid and gas argon, the pressure inside the cryostat and the contamination of the gas argon, as well as cameras to inspect the most critical detector elements. These systems and their performance are reviewed, and a few aspects of their future upgrade discussed.

40th International Conference on High Energy physics - ICHEP2020

July 28 - August 6, 2020

Prague, Czech Republic (virtual meeting)

\footnotetext{
${ }^{*}$ Speaker
} 


\section{Introduction}

The Deep Underground Neutrino Experiment (DUNE) is a new world-class particle physics experiment which aims to study several mysteries at the forefront of high energy physics, such as the matter-antimatter asymmetry of the Universe, the dynamics of supernova neutrino bursts or proton decay. It will be formed by three different components: (1) the most intense neutrino beam ever created, (2) a composite near detector installed right downstream of the neutrino source, and (3) a massive far detector situated $1.5 \mathrm{~km}$ underground. This far detector will be formed by four huge liquid argon time projection chambers (LArTPC) with an overall sensitive mass of $40 \mathrm{kt}$. This detector will be able to uniquely reconstruct neutrino interactions with image-like precision and unprecedented resolution [1].

Argon is a noble element, which liquefies at $88 \mathrm{~K}$. For this reason each of the four LArTPC's should be contained inside a complex cryostat. Since DUNE is expected to run for more than 10 years, and the interior of those cryostats will remain inaccessible during that time, it is crucial to monitor their state. Cryogenic instrumentation is a set of devices which aims at providing comprehensive monitoring and understanding of the cryostat, the cryogenics system (which provides LAr recirculation) and the detector, ensuring its long time reliability and providing input for the physics results [2].

Two large prototypes [3] of the DUNE far detector have been built and operated at the CERN Neutrino Platform from summer 2018 until summer 2020 in order to solve some remaining technical issues, test installation sequence, demonstrate long term operational stability and prove detector performance. One of the prototypes, ProtoDUNE-SP, uses the single-phase technology, and the other, ProtoDUNE-DP, the dual-phase technology. Almost all DUNE instrumentation, including cryogenic instrumentation, has been tested at ProtoDUNE.

Each of the ProtoDUNE modules consists in a LArTPC contained inside a cryostat whose inner dimensions are $8.548 \times 8.548 \times 7.900 \mathrm{~m}^{3}$, with an overall argon mass of 770 tons. They are connected to a complete recirculation system that keeps the LAr purity sufficiently high to detect particles. The conjunct of the cryostat, the TPC and the LAr recirculation system conforms a very complex system with interdependent variables that are very sensitive to any variation of the cryogenic conditions [3]. During the last two years, having this system under control has been the main objective of the cryogenic instrumentation, formed by purity monitors, temperature monitors, cameras, gas analyzers, pressure sensors and level meters.

\section{Purity monitors}

A fundamental requirement of a LArTPC is that ionization electrons drift over long distances in the liquid, such that most of them arrive to the anode. Part of the charge is inevitably lost due to the presence of electronegative impurities in the argon. To minimize the charge loss, LAr purification is essential. The purity monitors (PrM) are in charge of monitoring the LAr purity inside the cryostat.

A purity monitor is a miniature TPC placed inside the cryostat that measures the electron lifetime (and thus, the impurity concentration) in the LAr. It generates photoelectric discharges in the cathode by means of a UV light source [4]. Due to the electric field applied between anode and cathode, electrons travels towards the anode where they are collected. The loss of charge in the 
process can be parametrized as $Q_{\text {anode }}=Q_{\text {cathode }} * e^{-t / \tau}$, from where the electron lifetime, $\tau$, can be computed. The range of accessible values of $\tau$ depends on the electric field applied and the distance between anode and cathode (PrM length).

In the case of the SP module, a vertical array of three purity monitors of $25 \mathrm{~cm}$ length each was installed, each on of them at a different height inside the cryostat. In the case of the DP module, a vertical array of two purity monitors of $15 \mathrm{~cm}$ length was installed, one of them at the bottom of the cryostat and the other one two meters above. An additional longer purity monitor of $30 \mathrm{~cm}$ was also installed at the bottom of the DP module. Having purity monitors at different heights helps in understanding the relation between the location in the cryostat and the purity of the LAr.

During the two years of ProtoDUNE operation, purity monitors have provided a comprehensive monitoring of the LAr purity. Figure 1 shows how the electron lifetime grew from zero after the LAr filling of the cryostat and how it has stayed above $10 \mathrm{~ms}$ most of the time, comfortably fulfilling the DUNE $3 \mathrm{~ms}$ requirement [2],[5]. The several purity drops are related to problems in the recirculation system.

For more details about the purity monitors, see [6]
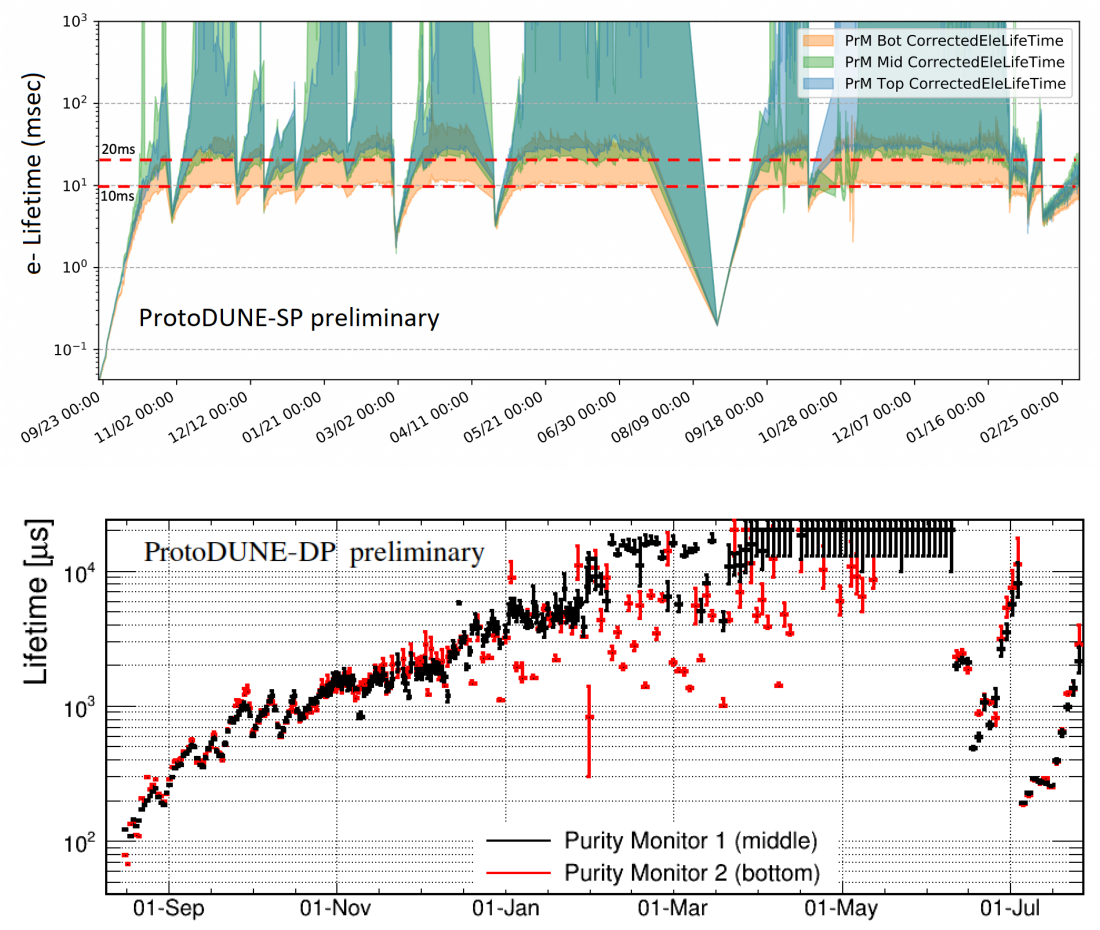

Figure 1: Top: Electron lifetime measured in the SP module since September 2018. Bottom: Electron lifetime measured in the DP module since September 2019.

\section{Temperature Monitors}

Complete monitoring of the temperature of the gas and liquid argon as well as of the cryostat membrane was achieved by the temperature monitoring system, formed by platinum RTDs of $100 \Omega$ 
resistance (PT1000) [3]. Its purpose was twofold. First, they were installed to monitor the cryostat cool-down and filling processes, both critical steps of the experiment operation since it is when the detector suffers the largest temperature gradients. Second (only in SP module), they have provided precise measurements of the LAr temperature in the cryostat, necessary to guarantee the correct LAr recirculation. It is known from previous prototypes that vertical temperature gradients of only $20 \mathrm{mK}$ can generate LAr stratification. For that reason these precise measurements (at $\mathrm{mK}$ level) are being used as an input for computational fluid dynamics (CFD) simulations, used to understand the LAr behavior in the cryostat and to predict the LAr purity throughout the active volume, later used for physics analyses [2].

Two vertical arrays with 24 and 48 temperature sensors respectively were installed in ProtoDUNESP [3]. The resulting temperature gradients as well as their comparisons with the CFD simulations are shown in Figure 2. A slightly larger gradient $(\sim 10 \mathrm{mK})$ close to the LAr surface can be seen in Figure 2, right. However, as this gradient is outside the active volumen of the TPC there is no risk of stratification. It is worth noting that the resolution achieved is sufficient to characterize the small vertical gradients observed. It is also important to remark the extraordinary performance of the cryostat and the cryogenics system since the vertical profiles have varied less than $3 \mathrm{mK}$ during more than one year of normal operation.

For more details about the the temperature monitors, see [7].
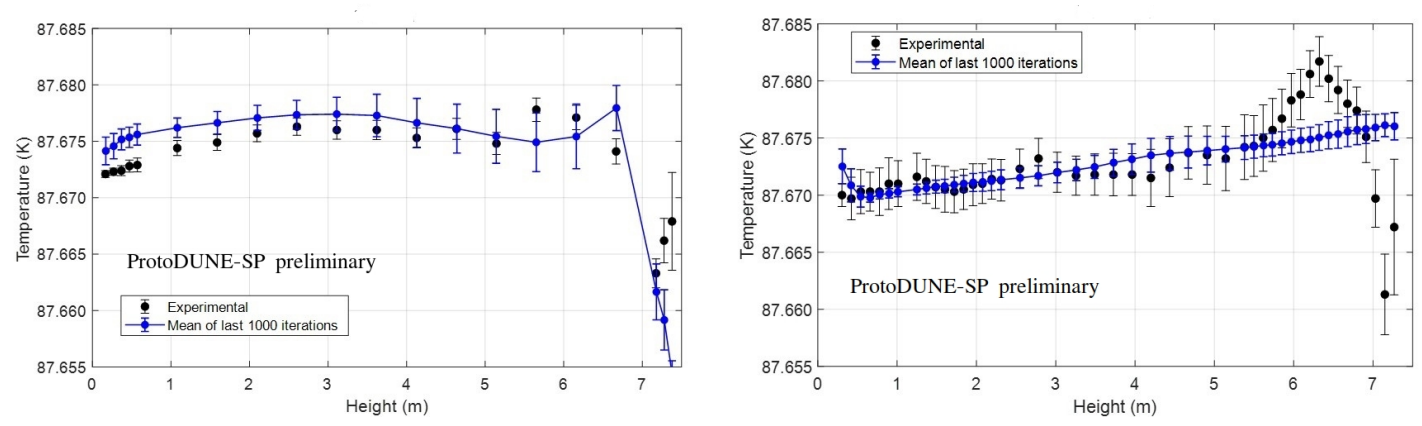

Figure 2: Comparative of the temperature gradient between data and simulations for both vertical arrays of ProtoDUNE-SP.

\section{Cameras}

Cameras provide direct visual information about the state of the detector module during critical operations and when damage or unusual conditions are suspected.

Eleven cameras were deployed in each of the ProtoDUNE modules [3]. Their objective has been to verify the stability, straightness, and alignment of the hanging TPC structures during cooldown and filling. Additionally, they have been used to inspect the movable parts of the experiments and to inspect the modules to look for the source of potential problems. For example, in the case of the SP module, they were used to look for sparks in the ground planes, and in the DP module they were used to understand the source of bubbles occurring near the charge readout planes.

Two types of cameras were installed. The so-called cold cameras were deployed in the liquid, inside rigid enclosures, to monitor the different parts of the detector during the cool-down and 
filling processes. In DUNE they are expected to survive at least the cool-down and filling phases, which will take about one year [2]. This has been proven in ProtoDUNE-SP, for which all cameras were operational after 1.5 years.

Warm cameras were installed in the ullage inside acrylic tubes that could be accessed from outside of the cryostat. They were designed to be as versatile as possible and for that reason they were reoriented several times. Additionally, one of them was completely replaced without contaminating the LAr. This reduces the requirements for the DUNE far detector in terms of durability [2].

\section{Gas analyzers}

Achieving and holding the required LAr purity demands a powerful cryogenic circuit capable of recirculating and purifying all the argon inside the cryostat, which in ProtoDUNE takes 5.5 days. The liquid is extracted from the bottom, purified and returned inside. The gas is extracted from chimneys at the top, liquefied, purified and returned as liquid. Gas analyzers sample the gas in those chimneys.

Gas analyzers are commercial hardware modules that measure the amount of contaminants in the argon gas. In the case of ProtoDUNE, they were used to measure traces of water and $\mathrm{O}_{2}$, required to be lower than $100 \mathrm{ppt}$, and traces of $\mathrm{N}_{2}$, required to be lower than $1 \mathrm{ppm}$. They successfully operated during the different stages of the experiment, being specially important during the filling process, in which the purity monitors were not completely sensitive to the impurity levels.

\section{Pressure sensors and level meters}

Pressure inside the cryostat and liquid argon level are two important variables from the physics point of view. The absolute value of the pressure sets the absolute temperature scale, which affects the electron drift velocity. Additionally, in the case of the DP module, the electron amplification in the change of phase depends on the gas pressure so it is extremely important to accurately measure it. Several differential and absolute pressure sensors were installed in both modules [3].

The liquid level measurement is important during LAr filling but also during operation. For the SP module the level should be always keep sufficiently above the ground planes, to avoid discharges. For the DP module it is essential to know at the sub-millimeter level the distance from the liquid surface to the readout plane, since the charge amplification factor depends on that distance. Two different types of level meters were installed: differential pressure level meters and capacitive level meters. The first ones use the pressure difference between their lower part, which is submerged in LAr, and their upper part, which is in the gas, and relate this pressure difference with the LAr level. These are generally used to measure the absolute level of LAr inside the cryostat. The second ones are empty capacitors whose capacitance depends on how much they are filled with LAr. These ones, much more sensitive to small LAr variations, are generally placed in the charge readout planes of the DP module, and have been proven to be sensitive to displacements of the order of $100 \mu \mathrm{m}$.

\section{Conclusions}

In order to not compromise physics results, comprehensive monitoring of the cryostat, the cryogenics system and the detector is necessary, both for the DUNE far detector and for their 
prototypes at CERN.

During the almost two years of operation of ProtoDUNE, its cryogenic instrumentation has substantially contributed to monitoring the state of the experiment, guaranteeing the integrity of several of its elements, its performance and ensuring its long term operational stability. All requirements have been comfortably fulfilled, demonstrating the robustness and performance of the cryogenics instrumentation devices.

Cryogenic instrumentation is being upgraded for the second run of ProtoDUNE, in 2022, which constitutes the last prototyping step before the construction of the DUNE's far detector. Several lessons have been learned from the first run and some improvements will be introduced in the cryogenic instrumentation devices.

\section{References}

[1] DUNE Collaboration, B. Abi et al., Deep Underground Neutrino Experiment (DUNE), Far Detector Technical Design Report, Volume I Introduction to DUNE, JINST 15 (2020), no. 08 T08008, [arXiv: 2002 . 02967].

[2] DUNE Collaboration, B. Abi et al., Deep Underground Neutrino Experiment (DUNE), Far Detector Technical Design Report, Volume IV Far Detector Single-phase Technology, JINST 15 (2020), no. 08 T08010, [arXiv: 2002 . 03010].

[3] DUNE Collaboration, B. Abi et al., The Single-Phase ProtoDUNE Technical Design Report, arXiv: 1706.07081.

[4] M. Adamowski et al., The Liquid Argon Purity Demonstrator, JINST 9 (2014) P07005, [arXiv: 1403.7236].

[5] DUNE Collaboration, B. Abi et al., First results on ProtoDUNE-SP liquid argon time projection chamber performance from a beam test at the CERN Neutrino Platform, arXiv:2007.06722.

[6] R. Diurba, Purity Monitoring at ProtoDUNE, These proceedings.

[7] R. Dharmapalan, Moveable Thermometer System in ProtoDUNE, These proceedings. 